\title{
Catastrophes naturelles: le projet de loi à l'étude en Italie
}

\author{
par Dario Gaverbi*
}

\section{Introduction}

Parmi les événements qui peuvent provoquer de très grands sinistres, les catastrophes naturelles représentent des situations de risque exceptionnelles.

Rappelons la typologie classique des sinistres les plus graves:

- celui qui frappe un seul intérêt ou un ensemble unitaire de biens, de type Piper Alfa, Anversa ou Pasadena, et qui provoque un très grand dommage à cause de l'importance énorme des valeurs en jeu;

- celui qui frappe une pluralité d'intérêts ayant comme origine le même événement, de type Talidomide, Daria ou Mireille, et qui provoque un très grand dommage du fait de l'accumulation d'une multiplicité de dommages.

Typologie de grands sinistres

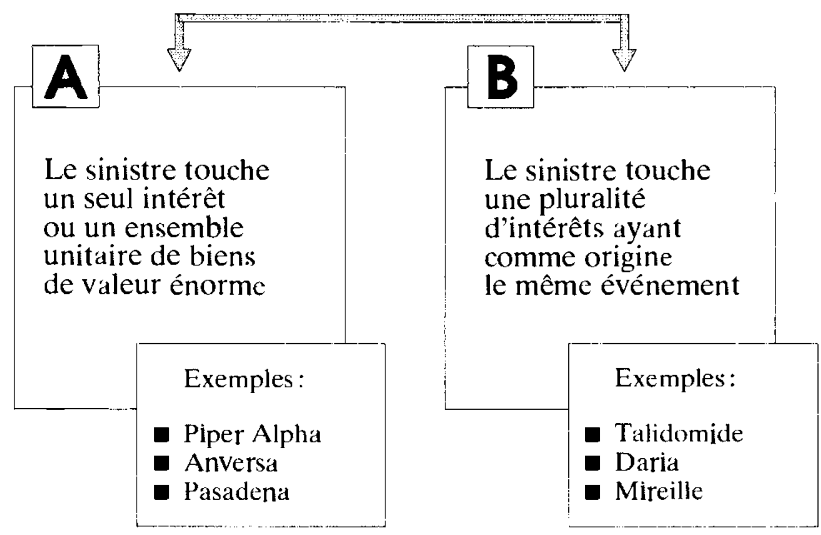

Les calamités naturelles jouent un rôle majeur parmi les causes des grands sinistres et revêtent des dimensions catastrophiques parmi ceux de type «B».

* Directeur Général, RAS, Milan. Texte présenté à Vienne à l'occasion de la 20ème Assemblée Générale de l'Association de Genève, le 7 juin 1993. 
Les catastrophes naturelles, comme d'autres événements catastrophiques, ont la propriétć de causer des dommages considérablcs, aussi bien par risque unique que par accumulation, en tenant comptc que cettc dernic̀re est depuis toujours la cause des plus grands désastres de notre monde.

En effet, les catastrophes naturclles pcuvent non seulement déstabiliser une entreprise, aussi grande soit-elle, mais également - dans le cas d'accumulation - l'économie même d'une région, si ce n'est d'un pays tout entier.

Parmi lcs autres nations europécnnes, l'Italie est particulièrement exposéc à ce genre d'événcments.

On n'à commencé à faire des statistiques sur les catastrophes naturelles ct les dommages causés par celles-ci qu'à la suite des dégâts très importants causés par lcs inondations dans le Polesinc au début des années 60, puis à Florence.

Les dépenses encourues entre 1968 et 1988 sont détaillées dans les tableaux qui suivent, et concernent les risques fondamentaux considérés comme des catastrophes naturelles.

\section{Typologie des événements catastrophiques}

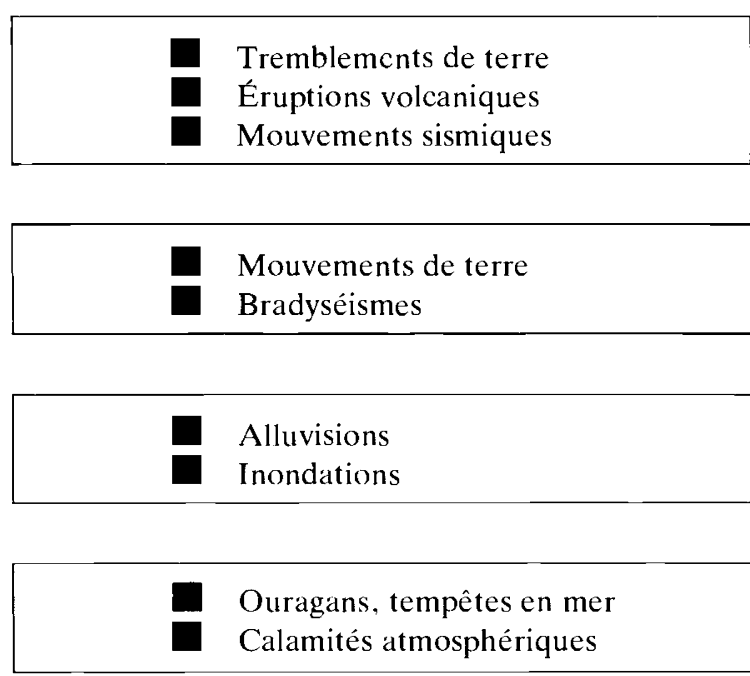

Entre 1968 et 1988, le total des dépenses effectuées par le gouvernement, pour faire face aux catastrophes naturelles survenues et reconnues se monte, en valeur actualisée, à environ 129.000 milliards répartis entre les quatre sections fondamentales précédemment indiquées. 
Tremblements de terre, éruptions volcaniques et mouvements séismiques

\begin{tabular}{|c|c|c|c|}
\hline Année & Localité & $\begin{array}{r}\text { Entité du dommage } \\
\text { (Milliards de Lires } \\
\text { réévaluées } \\
\text { au } 31.12 .92 \text { ) }\end{array}$ & $\begin{array}{r}\text { \$USA } \\
\text { (Millions) }\end{array}$ \\
\hline 1968 & VALLE DEL BELICE & 8.986 & 5.990 \\
\hline 1972 & MARCHE & 2.617 & 1.744 \\
\hline 1972 & ABRUZZO/UMBRIA & 481 & 321 \\
\hline 1975 & PERUGIA & 143 & 95 \\
\hline 1976 & FRIULI & 21.168 & 14.112 \\
\hline 1978 & CALABRIA/SICILIA & 509 & 339 \\
\hline 1978 & UMBRIA & 126 & 84 \\
\hline 1978 & IRPINIA SANNIO & 80 & 53 \\
\hline 1979 & UMBRIA/MARCHE & 2.502 & 1.668 \\
\hline 1980 & CAMPANIA/BASILICATA & 67.256 & 44.837 \\
\hline 1981 & SICILIA OCCIDENTALE & 419 & 279 \\
\hline 1982 & CAMPANIA/BASILICATA & 598 & 398 \\
\hline 1982 & UMBRIA & 323 & 215 \\
\hline 1983 & PARMA/REGGIO EMILIA & 358 & 238 \\
\hline 1984 & ITALIA CENTRALE & 2.151 & 1.413 \\
\hline 1984 & ZAFFERANA ETNEA & 169 & 112 \\
\hline 1985 & UMBRIA & 11 & 7 \\
\hline 1986 & SICILIA ORIENTALE & 21 & 14 \\
\hline \multirow[t]{2}{*}{1987} & ITALIA CENTRAL & 148 & 98 \\
\hline & & 108.066 & 71.741 \\
\hline
\end{tabular}

Classements de terrain et bradyseisme

1971

1976

1982

1983

1985

1986

1968

1970

1971

1973

1976

1977

1977

1978

1978

1983
POZZUOLI

LECCO

ANCONA

SONDRIO

VAL DI FIEMME

COMMUNE DI SENISE

Total partiel

Inondation

PIEMONTE

PROVINCIA DI GENOVA

PROVINCIA DI VITERBO

SICILIA/CALABRIA

TRAPANI/AGRIGENTO

PIEMONTE

NORD ITALIA

PIEMONTE/VALLE D'AOSTA

ABRUZZO

FRIULI/LOMBARDIA

\begin{tabular}{lrr} 
& 2.169 & 1.446 \\
& 84 & 56 \\
& 1.104 & 736 \\
& 222 & 148 \\
& 129 & 86 \\
Total partiel & 1.512 & 1.008 \\
\cline { 2 - 3 } & 5.220 & 3.480 \\
ndation & & \\
& 2.566 & 1.710 \\
& 585 & 390 \\
& 312 & 208 \\
& 2.379 & 1.586 \\
& 394 & 262 \\
& 334 & 222 \\
& 888 & 592 \\
Total partiel & 408 & 272 \\
& 35 & 23 \\
& 120 & 80 \\
\cline { 2 - 3 } & 8.021 & 5.345
\end{tabular}




\begin{tabular}{|c|c|c|c|}
\hline Année & Localité & $\begin{array}{r}\text { Entité du dommage } \\
\text { (Milliards de Lires } \\
\text { réévaluées } \\
\text { au } 31.12 .92 \text { ) } \\
\end{array}$ & $\begin{array}{r}\text { \$USA } \\
\text { (Millions) }\end{array}$ \\
\hline 1977 & BASILICATA & 933 & 622 \\
\hline 1978 & MESSINA/AGRIGENTO & 52 & 34 \\
\hline 1981 & CALABRIA/SICILIA & 241 & 160 \\
\hline 1985 & DISTRIB. SUL TERRITORIO & 1.395 & 930 \\
\hline 1987 & DISTRIB. SUL TERRITORIO & 1.963 & 1.308 \\
\hline \multirow[t]{2}{*}{1987} & VALTELLINA & 3.030 & 2.020 \\
\hline & & 7.614 & 5.074 \\
\hline
\end{tabular}

Total des dépenses effectuées de 1968 à 1988

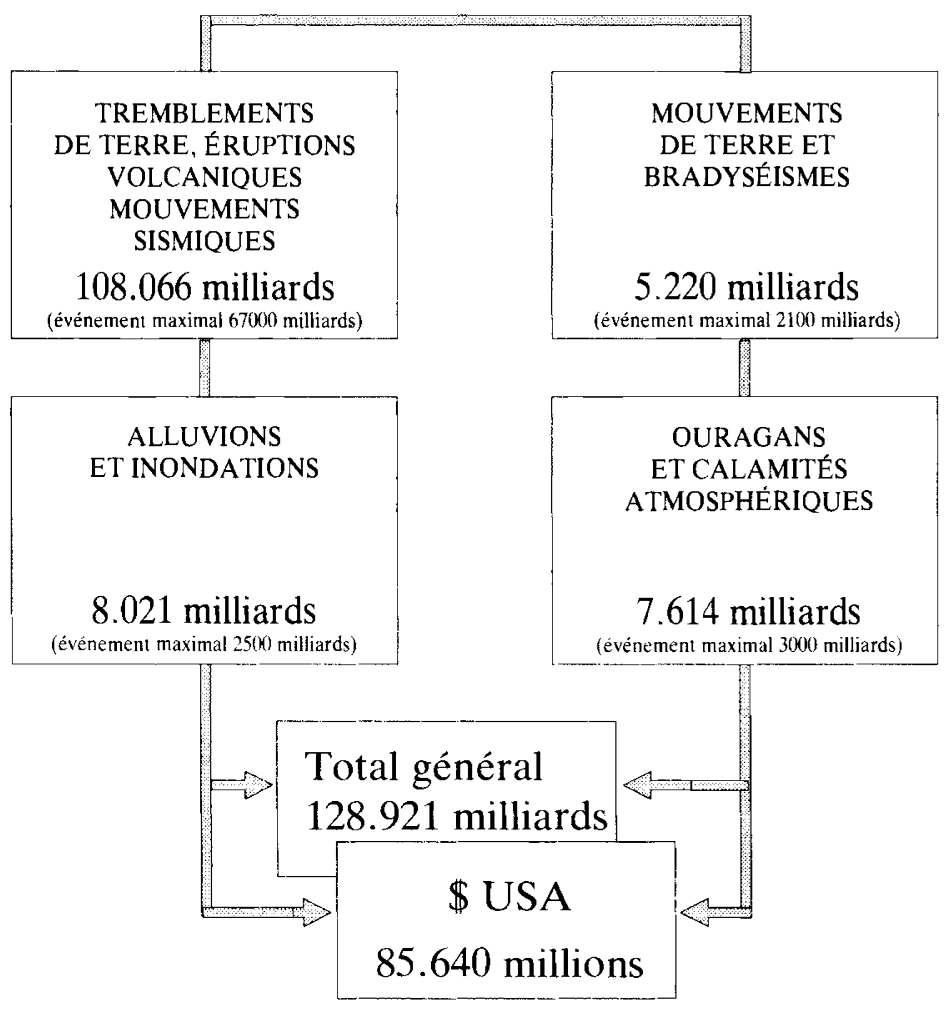

Vu le nombre de cas où ont été constatées de très fortes exagérations de dol pour les dommages subis, les organes gouvernementaux sont arrivés à la conclusion que la liquidation directe des dommages conduisait, en l'absence d'une documentation valable sur 
les biens préexistants et sur leur valeur, à des paiements non dus et incontrôlés. Il s'est alors agi de savoir si le marché de l'assurance était disposé à étudier le problème et à collaborer en vue de trouver une solution.

Une commission d'étude créée dans ce but, a défini les concepts essentiels et confirmé que les assureurs étaient tout à fait disposés à collaborer. Des choix se sont présentés :

1. choix entre le seul coût du contrôle du dommage et de sa définition, et la gestion intégrale du risque sous forme d'assurance avec prime et indemnisations ;

le choix s'est porté sur la gestion intégrale du risque par les assureurs, avec intervention de l'Etat en tant que réassureur.

2. dans le cas de gestion intégrale, choix entre système obligatoire et système facultatif; le choix s'est porté sur le système obligatoire.

Les alternatives des assureurs

\begin{tabular}{|l|l|l|l}
\hline $\begin{array}{l}\text { Le seul service } \\
\text { de la constatation } \\
\text { du dommage }\end{array}$ & ou bien & $\begin{array}{l}\text { La gestion intégrale } \\
\text { du risque, sous } \\
\text { forme d'assurance, } \\
\text { avec primes } \\
\text { et indemnisations }\end{array}$ \\
\hline
\end{tabular}

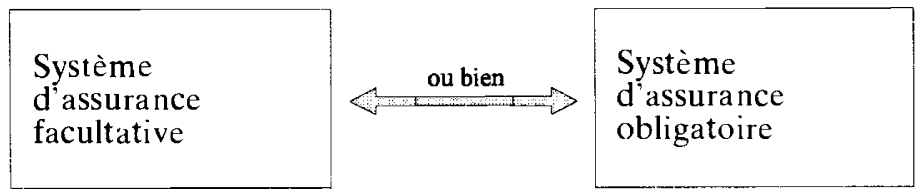

Le choix s'est porté sur la gestion intégrale du risque et l'assurance obligatoire.

\section{Raisons des deux choix}

L'hypothèse de seule définition et liquidation du dommage a été écartée, car :

- des pressions de toutes sortes et des phénomènes de clientélisme se seraient produits, avant ou après les contrôles;

— un contrat comportant des références précises n'existant pas, le dommage n'aurait pas eu une base préétablie pour sa définition;

- en ce qui concerne le coût du service, il aurait certainement été demandé aux assureurs de se contenter de très peu comme devoir social, ce qui aurait signifié soit une perte d'argent, soit une mauvaise réalisation du travail, accompagné de répercussions négatives sur l'image de marque;

- au contraire, les responsabilités auraient été énormes, et les contrastes entre les victimes de tremblements de terre ou d'inondations auraient été une source potentielle de conséquences très graves. 
La conclusion était qu'il fallait délimiter le système d'assurance dans les deux sens, celui de la «collecte» des contributions et celui du payement des indemnisations.

L'hypothèse du système facultatif a èté ćcartèe à cause de ses dẻfauts intrinsèques:

- aucun apport de solidaritè, car les non-exposès ne se seraient pas assurès;

- mutualité limitée à peu de risques;

- anti-sèlection poussèe au maximum;

— taux de prime très èlevès;

- difficultès insurmontables pour assurer ceux qui en auraient besoin.

En conclusion, le vrai problème de l'Etat italien n'ètait pas résolu.

Le choix final en faveur de l'obligation cst né de la réflexion qu'elle a génèré:

- une mutualitè suffisamment vaste,

- répondant aux fins d'une solidaritè nationale,

- basée sur le principe selon lequcl celui qui payc sa contribution a le droit, en cas de catastrophe naturelle, d'être indemnisé,

— une localisation facile pour pouvoir être remboursè facilement,

- rapport pour le contrôle et la liquidation des dommages selon les modalitès de l'assurance du contrat intèressé,

- prédisposition à èliminer le nœud central de l'anti-sèlection,

- soutien indispensable par une garantie de l'Etat,

- acceptation par le public du fait de la modicite de la contribution requise.

Les points fondamentaux du projet de loi actuellement examiné peuvent se résumer de la manière suivante:

1) obligation de l'assurance,

2) definition du terme «catastrophe naturelle»,

3) opérationnalitè de la garantie,

4) montant de la contribution ou de la prime,

5) délivrance de la police d'assurance,

6) limitation de la garantie et base de l'indemnisation,

7) intervention des experts,

8) modalitė du mécanisme de réassurance.

\section{Les caractéristiques du projet}

Le développement de ces huit points met en évidence les caractèristiques suivantes:

1) L'assurance obligatoire contre les dègâts provoquės par les catastrophes naturelles sur les biens de proprièté appartenant à des personnes physiques ou morales, en excluant ceux appartenant directement ou indirectement à l'Etat, existant sur le territoire de la République (meubles et immeubles ou seulement immeubles) avec une police d'assurance spècifique au but.

2) Les catastrophes naturelles sont, selon la loi, les:

- tremblements de terre,

- éruptions volcaniques, 
- inondations,

- tempêtes en mer,

- trombes ou autres tempêtes,

- glissements de terrain.

Leur définition est contenue dans cette même loi.

3) L'assurance fonctionne à condition que la valeur réelle des biens possédés ait été stipulée dans le contrat, et que la prime ait été versée à l'assureur qui a délivré la police.

4) La prime est calculée en fonction de la valeur déclarée des biens. Son montant est unique pour tout le territoire national selon le principe de solidarité prévu par la Constitution. Ce montant est établi par la réglementation législative et pourra être modifié si cela s'avère nécessaire.

5) La délivrance des polices d'assurance contre les dommages dus à des catastrophes naturelles est consentie à toutes les entreprises autorisées à opérer dans les secteurs 8 et 9 de la Loi 295 du 10 juin 1978.

6) La base d'indemnisation concerne les valeurs réelles des biens selon les déclarations du point 3 ) précédent, sujettes aux limitations ou paramétrisations fixées par le règlement, exception faite des dommages indirects.

7) Prédisposition, sous l'égide du Ministère de la Protection Civile, des accords nécessaires entre l'association des assureurs et celle des experts contrôleurs des dommages pour établir la procédure et assurer une gestion efficace de l'urgence et de la régulation des dommages.

8) Les entreprises d'assurance autorisées à délivrer des polices contre les dommages résultant de catastrophes naturelles conservent une quote-part des risques assumés, mais sont réassurées en quote-part et en excédent de pertes par un organisme de l'Etat.

En complément, le projet de loi réglemente les obligations inhérentes à la prévention, la déductibilité fiscale des contributions et les sanctions à charge des non-assurés.

Le projet, une fois choisis le système obligatoire et la gestion intégrale des risques sous forme d'assurance avec primes et indemnisations, s'est concentré sur deux points fondamentaux :

A) le premier concerne le coût prévisible de la couverture pour le public, même s'il s'agit d'une approximation, et donc son impact sur le marché,

B) le deuxieme traite du système de fonctionnement du mécanisme de réassurance et des contrôles techniques indispensables.

A) L'évaluation du coût formulé se base sur une série d'hypothèses raisonnables tenant compte:

1) du poids de la sinistralité réduisant le coût des indemnisations à travers l'adoption de limites maximales pour la couverture des patrimoines et l'application de franchises, même en tenant compte du fait que la certitude du remboursement fera augmenter l'entité des demandes.

Grâce à ce qui précède, il est possible d'estimer le coût total des catastrophes, entre 1968 et 1988 , à 100.000 milliards de lires, soit 500.000 milliards de lires en s'ajustant au cours du siècle. 
2) Le coût annuel de garantie d'assurance des catastrophes est, par conséquent, évalué à 5.000 milliards, équivalant au montant approximatif des contributions ou des primes, afin de faire face aux sinistres et aux dépenses.

3) L'entière valeur des biens obligatoirement assurés devrait s'élever à 20.000 .000 milliards dc lires, même s'il s'agit d'un chiffre très approximatif, calculé sur la base d'estimations.

4) Le taux de prime annuel unique résultant de la combinaison des facteurs cités aux points 2 ) et 3 ) s'élève environ à 0.25 pour mille, ce qui conduit à un coût de garantie de 50.000 lires pour un patrimoine familial de 200.000.000 lires de biens.

Si ce résultat est vérifié, ce coût pourrait être considéré comme acceptable pour le marché, compte tenu de la configuration et du taux de risque de l'Italie par rapport aux catastrophes naturelles.

\section{Synthese}

Hypothèse d'approximation large du calcul de la prime.

1) Réduction de la sinistralité par des limites maximales de couverture et par des franchises: par hypothèse, 500.000 milliards de lires au cours du sieccle, équivalant à une charge annuelle de 5.000 milliards.

2) Hypothèse de la valeur des biens obligatoirement assurés: 20.000.000 de milliards de lires.

3) Taux de la prime annuelle unique pour tous les biens de $0,25 \%$ correspondant à un coût de 50.000 lires pour un patrimoine familial de 200.000 .000 de lires.

B) Le système supposé de fonctionnement de réassurance adopte les mécanismes consolidés des techniques d'assurance et de réassurance, dont on peut définir les points principaux :

1) l'hypothèse de répartition du risque, qui se base sur trois capacités:

- la capacité nette totale des compagnies du marché italien, en tenant compte de l'importance des primes en jeu,

- la capacité additionnelle du marché mondial de la réassurance,

- l'absorption par l'Etat des niveaux de risque plus élevés.

Ces trois capacités fonctionnent comme trois couches superposées d'une capacité totale maximale par événement, réparties selon une juste proportion entre les coûts à supporter et les primes conservées.

2) L'éventualité d'un sinistre très supérieur ou supérieur à la capacité par événement concernée et placée à la base de la répartition devra faire l'objet d'une surveillance attentive pendant les premières années. 
Le projet prévoit l'absorption par l'Etat de ces sinistres durant les périodes initiales, l'Etat lui-même venant à fonctionner comme chambre de compensation pour ces dommages.

3) Une autre éventualité serait qu'il y ait une répétition des sinistres durant la même année, avant que les réserves d'équilibre n'aient été constituées.

Pour cela aussi, l'intervention de l'Etat est l'unique solution pour constituter des fonds nécessaires, par analogie à ce qui a été dit au point 2).

\section{Synthèse}

Hypothèses de règle générale ébauchée par le système de réassurance

1) Utilisation de la capacité par événement du marché italien et du marché mondial de réassurance, avec absorption par l'Etat des risques les plus élevés.

2) Protection du système par l'Etat contre l'éventualité de sinistres très graves, supérieurs à l'événement maximal durant la période initiale.

3) Protection analogue contre une éventuelle répétitivité de sinistres majeurs ou mineurs de l'événement maximal durant la période initiale.

Reste la question de savoir si les marchés internationaux seront disposés à aider le marché italien même dans l'hypothèse d'un équilibre final garanti.

Nous espérons que, si ce projet est développé et se finalise, il soit la preuve d'une Europe qui avance et d'un plus grand sens des responsabilités et de la solidarité.

\section{ANNEXE 1:}

\section{La directive Seveso et son application en Italie}

Le 24 juin 1982 fut promulguée la Directive CEE n. 82/501, appelée «Directive Seveso» parce que élaborée à la suite du sinistre de Seveso. Elle concerne la prévention d'accidents importants qui pourraient être causés par des activités industrielles spécifiques du secteur chimique.

Cette directive, qui aurait dû être intégrée dans la législation italienne au plus tard le 8 janvier 1984, ne fut mise en cuvre qu'avec le DPR n. 175 du 17 mai 1988.

Ce décret impose aux responsables d'une activité industrielle, faisant partie de celles énumérées dans ce même décret, une série d'obligations suivant le type d'activité (déjà existante ou nouvelle) et, pour chaque activité, suivant le type et la quantité des substances traitées, produites ou stockées. 


\section{Activités industrielles existantes}

Les responsables des activités industrielles existantes sont sujets à deux types d'obligations :

1) obligations de caractère général, consistant à déterminer les risques d'accidents importants et à adopter des mesures de sécurité aptes à en prévenir et à en limiter les conséquences pour l'homme et pour l'environnement, en respectant les dispositions et les normes en vigueur aussi bien en ce qui concerne la sécurité et l'hygiène du travail que la protection de la population et de l'environnement.

2) obligations spécifiques telles que:

- la notification, au ministre de l'Environnement et au ministre de la Santé, des activités exercées, accompagnée d'une série d'informations, y compris l'indication des éventuelles mesures d'assurance et de garantie adoptées pour les risques de dommage à la personne, aux choses et à l'environnement;

- déclaration à faire parvenir à la Région et au Préfet quant à l'utilisation, dans l'activité exercée, de substances dangereuses parmi celles énumérées, et l'éventuelle adoption de mesures d'assurance et de garantie par les risques de dommage à la personne, aux choses et à l'environnement;

- ajournement de la notification et de la déclaration sur la base des nouvelles connaissances en matière de sécurité et d'évaluation des risques.

\section{Nouvelles activités industrielles}

En outre, les responsables de telles activités doivent fournir aux ministres de l'Environnement et de la Santé une expertise assermentée qui atteste:

- la véracité et le caractère exhaustif des informations,

- la conformité des mesures de sécurité adoptées à celles établies par les prescriptions générales.

Le texte du DPR 175/88 ne contient aucun article qui se réfère de manière spécifique à la responsabilité du fabricant. En effet, il ne prévoit pas l'obligation de donner des garanties financières ou de stipuler des polices d'assurance pour dommage à la personne, aux choses et à l'environnement, mais se limite à demander au fabricant d'indiquer quelles mesures d'assurance et de garantie il a adoptées pour couvrir le risque de dommage à la personne, aux choses et à l'environnement en relation avec l'activité exercée.

Mais, au cours de ces dernières années, on a assisté à une aggravation notable des sinistres dûs à l'incendie et à l'explosion. Ce sont des dommages ou des événements semblables qui ont touché les activités industrielles rentrant dans le DPR cité précédemment.

Il y a eu de nombreux cas où les indemnités versées par les assureurs ont été partiellement ou totalement insuffisantes, soit parce que l'entreprise était sous-assurée, soit parce que le non-respect des normes de sécurité avait engendré une aggravation du risque.

Le moment semble donc venu d'établir, pour des activités industrielles déterminées, l'obligation d'être assuré pour les risques d'accidents majeurs, en plus de la vérification de la conformité des installations aux requêtes générales de sécurité prévues pour l'exercice d'activités à haut risque.

La garantie d'assurance devrait couvrir autant les biens de l'entreprise que les dommages qui, comme conséquence de ces événements, pourraient être causés à des tiers (personnes, animaux ou choses). 
Il est évident qu'une telle obligation, qui serait à instaurer (avec un amendement ct certains articles du DPR), ne pourrait qu'apporter d'indiscutables avantages au monde de l'assurance qui, cn conséquence, verrait son champ d'action s'ćlargir sensiblement.

\section{ANNEXE 2:}

\section{Directive «Environnement» et son application en Italie}

L'Evaluation de l'Impact sur l'Environnement (E.I.E.) est un procédé administratif qui vise à estimcr et à comparer, au préalable, les effets positifs et négatifs que des travaux d'une grande envergure peuvent produire sur l'environnement.

Les normes relatives à l'Evaluation de l'Impact sur l'Environncment ont comme origine la Directive CEE $85 / 337$ qui réglemente la procédure d'évaluation à laquelle doivent être soumis tous les projets publics et privés caractérisés par d'importantes conséquences pour l'cnvironnement.

En Italie, l'Evaluation de l'Impact sur l'Environnement est confiée à une procédure transitoire prévue par la Loi 8 juillet $1986 \mathrm{n}$. 349, qui a institué le Ministère de l'Environnement, à laquelle s'adjoignent deux autres DPCM du 10 août 1988 n. 377 et du 27 décembre 1988 et quelques normes régionales, d'où des problèmes conséquents de coordination pour l'attribution des compétences.

\section{La discipline communautaire}

La Directive CEE $85 / 337$ prévoit que certaines catégories de projets, par rapport auxquels l'importance de l'Impact sur l'Environnement est un objet de présomption absolue, doivent obligatoirement être soumis à la procédure de l'E.I.E.

Il s'agit des:

- raffineries,

- centrales thermiques,

- aciéries,

- installations chimiques intégrées,

- installations pour le stockage et l'élimination des déchets radioactifs, toxiques ou dangereux,

- autoroutes,

- ports commerciaux.

Par contre, d'autres types de projets, tels que les:

- installations d'extraction,

- industries textiles,

- industries papetières,

- industries métallurgiques,

- industries alimentaires,

- travaux de construction en général,

- dépôts, etc..,

caractérisés par un degré mineur d'impact sur l'environnement, ne peuvent former un objet de l'E.I.E. que si les Etats membres le jugent nćcessaire.

La procédure de l'E.I.E. prescrite par la Directive s'articule en quatre phases qui convergent vers la définition de compatibilité avec l'environnement. 
- La première phase consiste à présenter aux autorités compétentes des informations nécessaires à l'évaluation du projet;

- la dcuxic̀me phase prévoit l'élaboration des informations, à la charge des autorités compétentes;

- la troisième phase met en œuvre lc principe de la participation publique: les informations sont mises à la disposition de la collectivité, qui pcut exprimer son opinion au sujet de la réalisation du projet;

- dans la quatrième et dernière phase, les autorités compétentcs se prononcent sur la compatibilitć du travail avec l'environnement, appliquant ainsi la procédurc d'autorisation.

\section{La situation italienne}

S'ils avaient cxécuté la Dircetive 85/337, les Etats membres auraient dû adopter au plus tard lc 3 juillet 1988 les dispositions nécessaires afin que, avant la délivrance de l'autorisation, les projets concernant la réalisation de travaux comportant un impact majeur sur l'environnement - du fait de leur nature, leur dimension et leur cmplacement - soient soumis à I'E.I.E.

Jusqu'à présent, une procédure transitoire d'E. I. E. prévue par l'Article de la Loi 349, a été introduite dans le système italien, en attendant l'application complète de la directive.

L'obligation d'E.I.E. concerne essentiellement les nouvelles activités relatives aux catégories de travaux indiquées dans la directive. Elle s'étend également à deux typologies d'intervention sur des travaux déjà existants: celles qui altèrent radicalement les caractéristiques substantielles des travaux compris dans les catégories indiquées et celles qui modifient les travaux de manière à les ramener aux catégories déterminées par la directive.

Le jugement de compatibilité avec l'environnement, prononcé par le Ministère de l'Environnement - autoritć compétente en la matière - doit être formulé 90 jours après la communication du projet de la part du commettant. Après ce délai, la procédure d'approbation du projet reprend son cours normal.

Les DPCM successives du 10 août 1988 n. 377 et du 27 décembre 1988 dictent les normes techniques, et spécifient dans le détail quelles sont les catégories de travaux à soumettre à la procédure décrite ci-dessus, qui sont nouvelles, à l'exception de celles existant, en ce qui concerne les modifications survenues.

A la différence de la Loi Seveso, concernant les activités dangereuscs, la Loi 349 ne se réfère pas particulièrcment aux garanties d'assurance.

Notre conviction est, qu'ayant pris soin de l'importance des dommages possibles de type catastrophique, de la protection des biens et de la santé des citoyens, le concept d'obligation d'assurance devrait donner lieu à des garanties pour dommages et responsabilité, à partir du début de la construction des travaux entrant dans le cadre de la loi. 